\title{
Battlefield Cytoskeleton: Turning the Tide on Plant Immunity
}

\author{
Pai $\mathbf{L i}^{1,2}$ and Brad Day ${ }^{2,3, \dagger}$ \\ ${ }^{1}$ Department of Plant Biology; ${ }^{2}$ Department of Plant, Soil and Microbial Sciences, Michigan State University, East Lansing, MI \\ 48824, U.S.A.; and ${ }^{3}$ Michigan State University Plant Resilience Institute, East Lansing
}

Accepted 23 August 2018.

The plant immune system comprises a complex network of signaling processes, regulated not only by classically defined immune components (e.g., resistance genes) but also by a suite of developmental, environmental, abiotic, and biotic-associated factors. In total, it is the sum of these interactions-the connectivity to a seemingly endless array of environments-that ensures proper activation, and control, of a system that is responsible for cell surveillance and response to threats presented by invading pests and pathogens. Over the past decade, the field of plant pathology has witnessed the discovery of numerous points of convergence between immunity, growth, and development, as well as overlap with seemingly disparate processes such as those that underpin plant response to changes in the environment. Toward defining how immune signaling is regulated, recent studies have focused on dissecting the mechanisms that underpin receptor-ligand interactions, phosphoregulation of signaling cascades, and the modulation of host gene expression during infection. As one of the major regulators of these immune signaling cascades, the plant cytoskeleton is the stage from which immune-associated processes are mobilized and oriented and, in this role, it controls the movement of the organelles, proteins, and chemical signals that support plant defense signaling. In short, the cytoskeleton is the battlefield from which pathogens and plants volley virulence and resistance, transforming resistance to susceptibility. Herein, we discuss the role of the eukaryotic cytoskeleton as a platform for the function of the plant immune system.

\section{PLANT IMMUNITY}

The primary function of the plant immune system is to restrict pathogen invasion and multiplication, thereby inhibiting disease and death. At the same time, the immune system must also be regulated such that beneficial interactions are not negatively impacted (Tóth and Stacey 2015), as well as to permit plant growth and development (Huot et al. 2014). In both cases, the immune system plays a key role in how plants respond to the environment. Research in the area of plant-pathogen interactions has led to a model which describes two primary

${ }^{\dagger}$ Corresponding author: Brad Day; E-mail: bday@msu.edu

Funding: Research from the Day lab summarized herein was supported by grants from the U.S. National Science Foundation Division of Integrative Organismal Systems (IOS-1557437) and the National Institute of General Medical Sciences (1R01GM125743) to B. Day.

(c) 2019 The American Phytopathological Society nodes of the immune system (Chisholm et al. 2006). In short, these separate yet coordinately regulated pathways are defined, in large part, by the source and amplitude of the immuneeliciting signal (Jones and Dangl 2006). The first, referred to as pathogen-associated molecular pattern (PAMP) (e.g., flagellin, chitin, and lipopolysaccharide)-triggered immunity (PTI), is characterized by a rapid signaling response activated following the perception of conserved pathogen molecules by hostderived pattern recognition receptors (PRR) (Peng et al. 2018; Tang et al. 2017). In short, PTI results in the rapid activation of immune-associated signaling processes, including the generation of reactive oxygen species (ROS), the induction of second messenger signaling (e.g., $\mathrm{Ca}^{2+}$ ), and changes in gene expression ( $\mathrm{Li}$ et al. 2016).

As a counter response to the activation of PTI, many pathogens of plants deliver effector proteins into host cells to interfere with, or block, this initial immune signaling process (Büttner 2016; Lo Presti and Kahmann 2017). This, in turn, can lead to the induction of an enhanced immune signaling response referred to as effector-triggered immunity (ETI). This node of immunity is mediated by the activity of nucleotidebinding leucine-rich repeat (NB-LRR) resistance $(\mathrm{R})$ proteins which are activated following the recognition of delivered pathogen avirulence effectors ( $\mathrm{Su}$ et al. 2018). Converse to PTI, ETI results in a sustained immune response, which typically manifests in the induction of the hypersensitive response (HR) - programed cell death (PCD) — which is hypothesized to function in the restriction of pathogen growth (Huysmans et al. 2017).

\section{THE CYTOSKELETON AS A MOLECULAR AND CELLULAR SCAFFOLD OF PLANT IMMUNITY}

Two major classes of the plant cytoskeletal network are found in higher eukaryotes (Fig. 1). The first, microfilaments (MF), commonly referred to as the actin cytoskeleton, are formed by the polymerization of globular $(\mathrm{G})$-actin into filamentous $(\mathrm{F})$-actin, a process in plants that requires the function of more than 75 actin-binding proteins (Fig. 1A) (Day et al. 2011). Actin is responsible for functions ranging from cytoplasmic streaming (e.g., movement of organelles) and cell division to trafficking and endocytosis. The second, microtubules (MT), are composed of a complex array of $\alpha / \beta$-tubulin heterodimers, a network that is typically associated with cell growth and long-distance intercellular movement and communication (Fig. 1B) (Brandizzi and Wasteneys 2013). Both MF and MT exhibit a remarkable degree of rapid, seemingly random yet highly specific dynamism, represented by tremendous rates of polymerization and depolymerization. Together, these patterns 
of cytoskeletal organization yield a highly dynamic and tightly regulated framework that connects the intercellular components of the cell to an endless suite of microenvironments and physiological processes. The eukaryotic cytoskeleton engages a variety of signaling events, including those associated with cell division and development, organelle movement, vesicle trafficking, and immunity (Elliott and Shaw 2018; Porter and Day 2016). As a function of the plant immune system, an abundance of data supports roles for the cytoskeleton in at least two key aspects of the immune response: (i) establishment and maintenance of signaling-competent microenvironments and (ii) cellular trafficking (organelle, proteins, and small molecules). Below, we highlight current research in each of these areas, discussing the role of each of these in immunity and the function of each as linkages between immune signaling and the dynamism of the host cytoskeleton.

In a typical plant cell, the vast majority of the cytoskeleton stretches from the cytosol to attachment points at or near the plasma membrane (PM). This is significant, because the PM is regarded as one of the key signaling interfaces between the host and pathogen, supporting the function of two primary classes of immune receptors: PRR complexes and the coiled-coil type NB-LRR (CC-NLR) resistance proteins. Thus, it is not surprising that the cytoskeleton-PM interface is also a key component of the signaling processes associated with receptor activation, mobilization, and signaling transduction. Indeed, as a scaffold for many of these PM-associated processes, recent work has revealed that the plant cytoskeleton selectively interacts with-either directly or indirectly-numerous

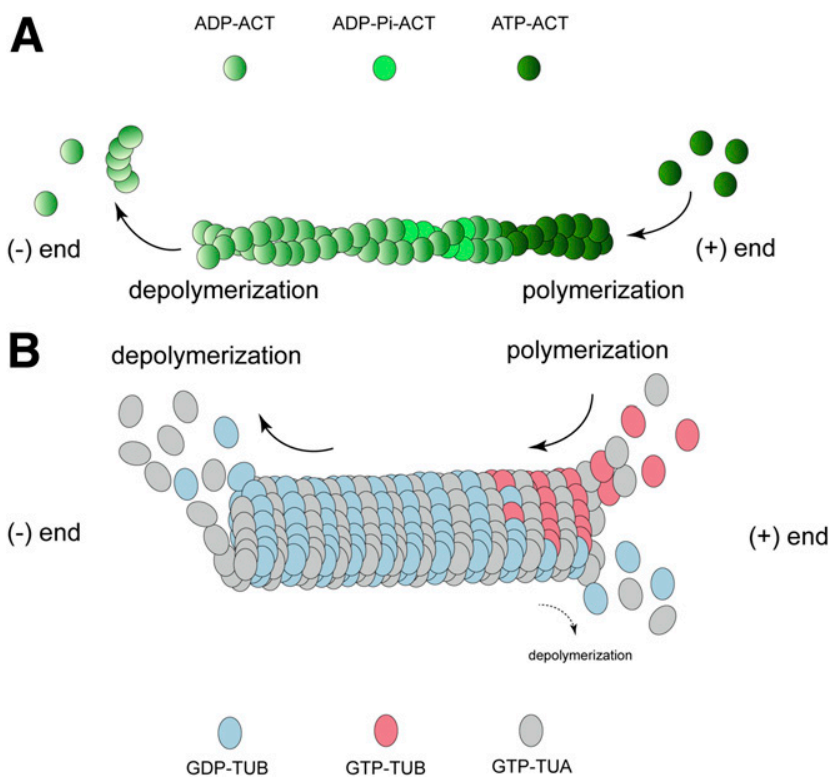

Fig. 1. Schematic diagram of cytoskeleton polymerization and depolymerization (treadmilling). A, Microfilament treadmilling. G-actin is dynamically polymerized onto the growing F-actin strand. The $(+)$ end is defined as the site where polymerization dominates and the (-) end as where depolymerization dominates, while spontaneous polymerization or depolymerization may occur on both sites. Actin polymerization is achieved through loading ATP-associated G-actin to the end of F-actin, while depolymerization occurs through destabilization of ADP-associated actin. $\mathbf{B}$, Microtubule treadmilling. The (+) end is defined as the site where both polymerization and depolymerization are very active, with polymerization dominating. Conversely, on the (-) end, tubulin is relatively stable with dominant depolymerization occurring. $\alpha$-Tubulin (TUA) and $\beta$-tubulin (TUB) form a heterodimer as the basic unit of the polymerized microtubule. TUA is constitutively bound to GTP; TUB binds the growing microtubule filament as GTP-bound monomers, and tends to disassociate from the filament when bound to GDP. membrane-localized receptors associated with immunity and signaling plant defense.

In Arabidopsis, the PM-localized PRR FLS2 (flagellin receptor) and BRI1 (brassinosteroid receptor), interact with BIK1 to form a coreceptor complex to initiate downstream signaling (Couto and Zipfel 2016). Following ligand binding, activated PRR complexes aggregate into distinct nanodomains within the PM, where they function in immune signaling activation (Keinath et al. 2010). Indeed, a recent study demonstrates that FLS2-BIK1 and BRI1-BIK1 complexes localize in distinct nanodomains within the PM, where they further associate with different proteins required for downstream signaling (Bücherl et al. 2017). In the case of BRI1-BIK1, the nanodomain has been shown to specifically interact with the MT network. This finding is significant because it provides experimental evidence that plant receptor kinases, including immune receptors, form functional complexes with the plant cytoskeleton to activate downstream signaling associated with immunity. In an additional study, it was further demonstrated that disruption of actin filament organization leads to the generation of a relatively enhanced ROS burst response following flg22 perception by FLS2 (Sun et al. 2018). In total, these studies were among the first to provide evidence supporting the hypothesis that cytoskeletal organization-and the physical interactions between PRR complexes and actin-are required for maintenance of appropriate levels of immune activation and signaling.

Although conclusive data demonstrating that the plant cytoskeleton directly interacts with individual immune receptors is lacking, an abundance of data in mammalian systems does exist. For example, the nucleotide-binding oligomerization domain protein 1 (NOD1), the PRR responsible for perception of p G-d-glutamyl-meso-diaminopimelic acid, requires F-actin for proper PM localization. Further, the interaction between NOD1 and actin serves as an immune interface which influences actin-remodeling and control of downstream signaling (Kufer et al. 2008), including the phospho-dependent activation of the actin depolymerizing factor cofilin (Bielig et al. 2014). Similar to the activation of NOD1, the mammalian muramyl dipeptide receptor NOD2 is also recruited to the PM through its association with actin (Legrand-Poels et al. 2007). Using a pharmacological-based approach, these studies also demonstrated that, following application of cytochalasin-D, an inhibitor of actin polymerization, both NOD1 and NOD2 signaling are activated, providing strong support for the hypothesis that the actin remodeling (including depolymerization) of PM-associated F-actin is likely a physical trigger of NOD1 or -2 signaling. Taken together, data from both plant and animal systems support the hypothesis that the cytoskeleton provides the necessary microenvironment to sustain the functionality of immune receptor complexes (Fig. 2 (a)) and, based on this, we hypothesize that the actin cytoskeleton is a guardee of PMlocalized PRR.

\section{THE ACTIN CYTOSKELETON IS REQUIRED FOR TURNOVER OF PM-LOCALIZED PRR}

During both PTI and ETI, the turnover of activated signaling complexes is mediated by receptor endocytosis, a process that functions not only to protect the plant from constitutive activation of defenses (i.e., autoimmune response) but also to support the surveillance function of the immune system $(\mathrm{He}$ et al. 2017). In the case of the PTI, recycling of PM-associated immune components is controlled in large part by clathrinmediated endocytosis (CME), a process that requires the function of the actin cytoskeleton (Nagawa et al. 2012). In wellstudied animal and yeast models, CME is initiated by loading the clathrin coat onto the PM components (e.g., PRR), which 
induces concomitant physical changes in the PM endocytic membrane fraction. Once the clathrin coat is loaded onto the cargo, the newly formed compartment gradually bends toward the cytosol, ultimately resulting in a scission from the membrane. Although the initial bending force that curves the membrane is provided by the clathrin-coated vesicles themselves, the growth and bending of the cargo-containing fraction is driven by actin polymerization. In short, this process is facilitated by the specific attachment of actin to the clathrin coat. Upon binding, the actin filaments extend by polymerizing and branching, a process mediated by the Arp2/3 complex and PM- associated myosin. This process is referred to as actin flow (Kaksonen and Roux 2018). It is predicted that plants utilize functionally and mechanistically analogous processes to those in animal systems (Fig. 2 (c)) yet, in plants, the actin branching structure required for actin flow may not be mediated by the Arp2/3 complex (Fan et al. 2015). This hypothesis is supported by data showing that the Arabidopsis Arp2/3 mutant does not have exhibit developmental lethality (Li et al. 2003), the expected phenotype if CME is fully inhibited.

In the case of plant immunity, multiple PRR, as well as numerous additional PM-associated proteins, have been

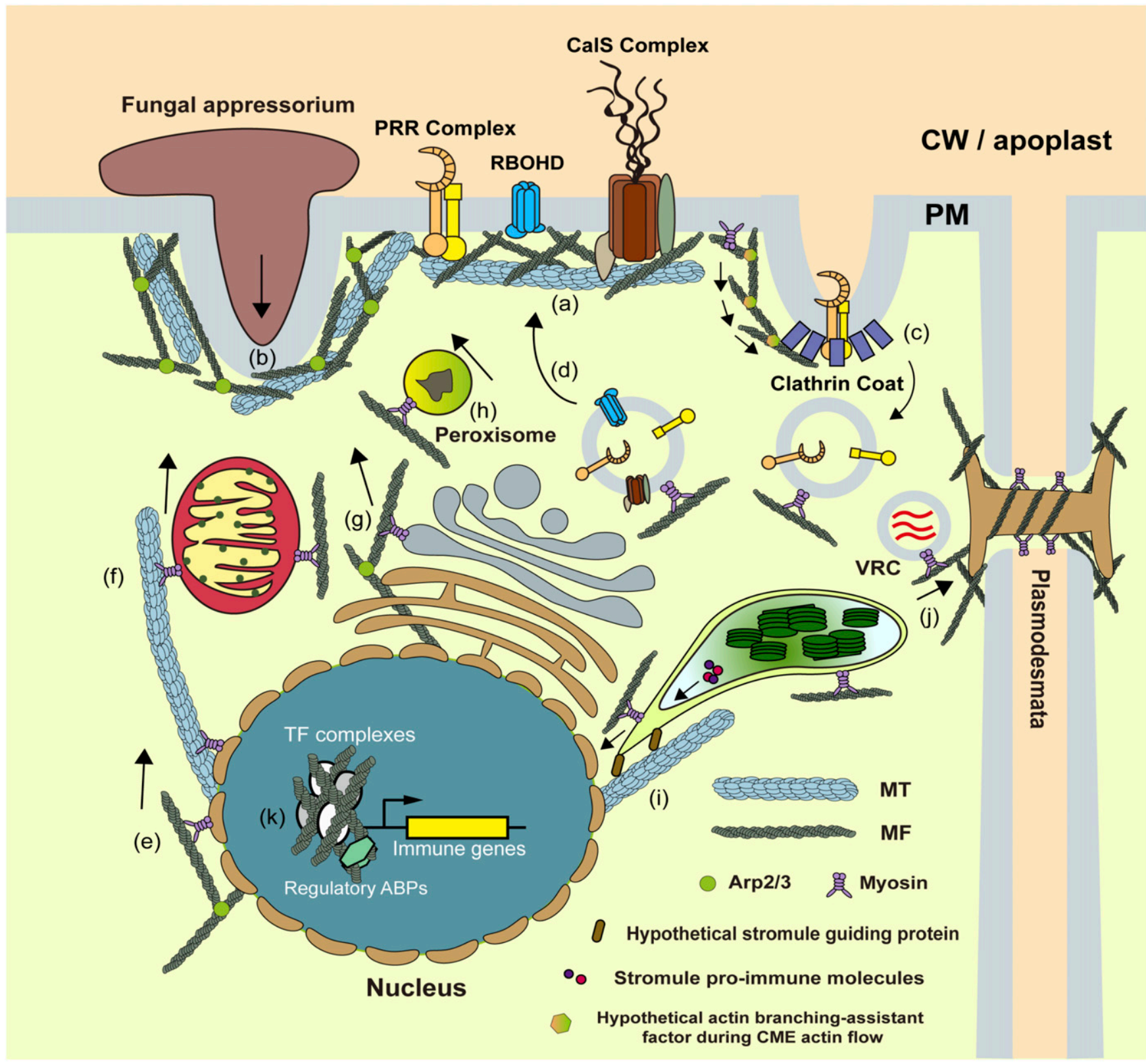

Fig. 2. The versatility and involvement of the plant cytoskeleton in immunity. Plant microfilaments (MF) and microtubules (MT) are involved in multiple processes during the immune response. (a) The cytoskeleton provides the physical attachment, as well as specialized microenvironments, to numerous plasma membrane (PM)-associated immune processes (e.g., pattern recognition receptor [PRR] complexes, RBOHD, and callose synthases [CalS] complexes) and is required for full functionality of these immune processes. (b) The cytoskeleton aggregates at the interaction interface of fungal pathogen penetration, a process that is even more striking in avirulent strains. The cytoskeleton is also required for proimmune cellular trafficking, a process that is associated with the transport of immune-functional molecules, through the action of (c) endocytosis (CW = cell wall); (d) PM and apoplast secretion; (e), (f), (g), and (h) transport of organelles; and (i) plastid stromules, as well as cell-to-cell trafficking through plasmodesmata. (j) Virus replication complex (VRC) can hijack cytoskeleton and transports to adjacent cells through plasmodesmata. (k) As a less-characterized mechanism of plant immunity, actin is also involved in the transcriptional regulation of immune signaling events within the nucleus, potentially through aiding in the formation of a regulatory complex consisting of transcription factors and chromatin. Arrows indicate the directionality or movement of corresponding cellular components. 
demonstrated to require CME for plant defense activation and signaling. For example, in the case of PTI, Mbengue and associates (2016) demonstrated that FLS2, elongation factor-Tu receptor (EFR), and pep1 receptor (PERP1/2) require clathrin, as well as the activity of BRI1-ASSOCIATED KINASE 1 (BAK1), for endocytosis, which are activated by corresponding PAMP. A second study further indicates that CME is required not only for the endocytosis of PEPR1 itself but also for the activation of PEPR1-mediated defense responses (Ortiz-Morea et al. 2016). Interestingly, myosin inhibitor 2,3-butanedione monoxime was found to inhibit FLS2 endocytosis, while the actin filament modifier latrunculin-B was shown to have only a minor impact on FLS2 endocytosis (Beck et al. 2012). Taken together, these data support a role for actin cytoskeletonmediated CME in the turnover and regulation of PM-associated immune receptors and their associated signaling processes. As one might expect, ETI-associated receptors also rely on CME for proper activity, as is the case for the tomato R protein $\mathrm{Cf}-4$, which functions in immunity against the pathogenic fungus Cladosporium fulvum (Postma et al. 2016).

\section{THE ROLE OF THE CYTOSKELETON IN INTERCELLULAR TRAFFICKING OF IMMUNE-ASSOCIATED PROCESSES}

The plant immune response relies on specialized patterns of cellular trafficking to deploy the suite of proteins, organelles, and small molecules required for pathogen resistance signaling (Park et al. 2018). To facilitate the rapid relocalization of immune components to the site of infection, both MF and MT are required for the specific trafficking of immune cargo to the site of infection (Brandizzi and Wasteneys 2013; Nebenführ and Dixit 2018; Tominaga and Ito 2015). Because a broader function underpinning the regulation of this process and, moreover, the connectivity to PTI, numerous studies have demonstrated that the plant immune signal involves the positive feedback in the expression of PM cell wall (CW)-associated immune components (Fig. 2 (d)), which include various signaling complexes, CW-associated polysaccharide synthases, and CW polysaccharide components synthesized in Golgi (Bacete et al. 2018; Schneider et al. 2016; van de Meene et al. 2017). For example, flg22 perception enhances the transcription of FLS2, $E F R, B A K 1$, and $R B O H D$ ( $\mathrm{Li}$ et al. 2016), a process that is hypothesized to compensate for the turnover (i.e., endocytosis) of PM-associated immune components to sustain the immune (i.e., PTI) signaling capacity of the cell. The enhanced expression of these PM-CW localized immune regulators requires a robust cytoskeleton system for their transportation and localization to the membrane. For instance, once pathogen signals (i.e., PAMP) are perceived, callose-enriched papillae between the CW and PM will form to inhibit pathogen penetration, which is regulated by salicylic and jasmonic acid pathways (Luna et al. 2011; Yi et al. 2014). At a mechanistic level, callose deposition requires callose synthases (CalS), enzymes that are sorted in Golgi and translocated to the CW. This process requires the activity of both MF and MT, and disruption of either cytoskeletal network leads to a dysfunction in CalS (Cai et al. 2011). Accordingly, in another study, it was demonstrated that an Arabidopsis class XI myosin mutant, with disrupted MF/MT trafficking, has dampened callose and lignin accumulation at the fungal infection site (Yang et al. 2014). Thus, from perception of PAMP to the activation of PTIassociated defense responses, the cytoskeletal network plays a key role in surveillance, activation, and the execution of immunity.

As noted above, the cytoskeleton is also required for the rapid relocalization of various host organelles and proteins to the site of pathogen penetration, a process that is hypothesized to enhance the immune response (Fig. 2 (e) to (h)). In one of the best-characterized examples, Takemoto and associates (2003) observed that the accumulation of Arabidopsis endoplasmic reticulum (ER) and Golgi occur at the infection site of oomycete plant pathogen Hyaloperonospora arabidopsidis, simultaneously with rapid remodeling of actin filaments. Subsequent work further showed that these events paralleled the redistribution of the host nucleus, ER, Golgi, mitochondria, and peroxisome at sites adjacent to penetration events during powdery mildew infection (Takemoto et al. 2006; Yang et al. 2014). We posit that these processes function to accelerate defense-associated metabolism, yielding an increase in the rate of response during infection via cytoskeletal-mediated cellular trafficking.

The recent discovery of a role for chloroplasts in plant immunity illustrates the complex relationships between immune signaling and the cytoskeletal network. As a component of the plant defense system, the chloroplast plays a role in the activation of HR-PCD through its degradation, which functions as a source of ROS burst following ETI elicitation (Dong and Chen 2013). Interestingly, disruption of the MT network has been shown to trigger chloroplast autophagy yet this same disruption attenuates cellular autophagy (Wang et al. 2015). Based on this, it is difficult to discern a role for the concomitant regulation of chloroplast and cytoskeleton as a function of HR-PCD. However, the explanation may lie in recent data describing the function of stromule formation during the activation of plant defense. A recent study found that chloroplasts form a tube-like architecture, called stromules, which stretches toward chloroplasts as well as other plastids and even the nucleus to mediate immune signaling (Fig. 2 (i)) (Hanson and Hines 2018). As an ETI-associated process, stromules were demonstrated to function in the transport of the N-receptor interacting protein 1 (Caplan et al. 2008) and potentially other proimmunity molecules into the nucleus to trigger the ETI against Tobacco mosaic virus effector p50 (Caplan et al. 2015). As a link to the engagement of the cytoskeleton, two recent studies confirmed that the extension of stromules from the chloroplast is mediated by the cytoskeleton (Erickson et al. 2018; Kumar et al. 2018). In brief, these studies demonstrate that MT guides the stromules for extension, and application of the MT-disrupting agents amiprophos-methyl or oryzalin inhibited the growth of stromules. In parallel, MF serves as the anchor point rather than the extension track (Kumar et al. 2018), potentially through binding of the stromule via class XI myosin (Natesan et al. 2009). Taken together, these studies provide compelling evidence indicating that the deployment of organelles and the transportation of their products is crucial for immune regulation, which relies on the activity of the cytoskeleton.

\section{BATTLEFIELD CYTOSKELETON: THE FRONTLINE OF PLANT DEFENSE AND PATHOGEN VIRULENCE}

Recent data from a suite of studies demonstrate numerous important roles for the plant cytoskeleton in the activation and signaling of plant immunity. However, questions remain: Is the reorganization of the cytoskeleton a response or a consequence? Is it associated with the activation of immunity or a process manipulated by pathogens to induce susceptibility? The short answer is both. A leading hypothesis in the field of cell biology and immunity is that the rapid and seemingly random reorganization of the cytoskeletal network is a plant-regulated cellular response to support immune signaling and downstream signaling of defense (Day et al. 2011). In this case, recent data 
demonstrate that pathogens alter both types of cytoskeletal structures during infection to evade immunity and promote infection. In the case of the former, recent work has shown that rapid changes in cytoskeletal organization occur during immune activation. For example, perception of the PAMP flg22, elf26, and chitin have all been shown to trigger the reorganization of actin in Arabidopsis epidermal cells (HentyRidilla et al. 2013, 2014) and in stomatal guard cells (Shimono et al. 2016a). As predicted, these PAMP-stimulated events require the PRR FLS2, EF-Tu, and CERK1, reinforcing the requirement of the actin cytoskeleton for PRR-PAMP function. Upon infection of tobacco BY-2 cells, the Pseudomonas syringae DC3000 type III secretion system (T3SS) helper protein, HrpZ, has been demonstrated to function as a PAMP, the perception of which induces bundling of F-actin and a concomitant decrease in MT density (Guan et al. 2013).

Alternatively, it is also demonstrated that pathogens can alter actin cytoskeletal structures during infection to evade immunity and promote infection. In a follow-up infection assay using $P$. syringae DC3000, it was observed that, although the MT architecture did not change within $16 \mathrm{~h}$ postinoculation (Lee et al. 2012); treatment for longer periods (i.e., $>20 \mathrm{~h}$ ) tended to induce long-term and multiple-phase influences on host actin. These changes included an initial increase in MF density, followed by a decrease in MF density, with a concomitant increase in MF bundling at later stages of infection (Henty-Ridilla et al. 2013). Importantly, a T3SSdeficient, avirulent, strain DC3000 $\Delta h r p H$ was unable to trigger the second phase of remodeling, suggesting a role of pathogen virulence by the T3SS as well as type III effectors (T3E) themselves (Shimono et al. 2016b).

In the case of fungal pathogens, similar to bacteria, avirulent and virulent strains confer differences in the pattern of cytoskeleton reorganization, illustrating a role for the cytoskeleton as a common immune component in response to multiple types of pathogens. In the well-defined barley-powdery mildew interaction system, avirulent strains will trigger the rapid reorganization of host MF and MT during the invasion process (Kobayashi et al. 1992; Miklis et al. 2007; Opalski et al. 2005); this response is indicated by actin bundling at the interface of the mature appressorium, with the formation of a dense network of MF surrounding the papillae. Such phenomena are referred to as actin focusing, with F-actin linking the host nucleus and the host-appressorium interface. For virulent strains, however, this pattern of filament organization is not observed, with only a slight aggregation of filament bundles without actin focusing (Kobayashi et al. 1992; Miklis et al. 2007; Opalski et al. 2005). Interestingly, MT remodeling patterns show a similar trend, with the induction of thick radial arrays of MT bundles at the site of appressorium formation in the presence of avirulent isolates and no aggregation in the presence of virulent strains (Kobayashi et al. 1992). Similar to powdery mildew, studies in the cowpea-rust fungi interaction system also demonstrated that avirulent strains trigger MF and MT reorganization, leading to a reduction in filament density, whereas no significant reorganization is observed in cells infected by virulent strains (Skalamera and Heath 1998).

In the case of bacterial pathogen infection, this phenomenon can be phenocopied by the application of cytoskeletal agents that interfere with MF and MT dynamics, manifesting in differing immune phenotypes between bacterial and fungal pathogens. For example, in the case of bacterial phytopathogens, disrupted MF increases resistance, including both PTI and ETI branches (Henty-Ridilla et al. 2013; Kang et al. 2014; Krutinova et al. 2018; Tian et al. 2009), whereas disrupted MT increase susceptibility to infection (Lee et al. 2012). However, host resistance to fungal pathogens is usually dampened by both MF and MT dynamics inhibitors (Schmidt and Panstruga 2007). These data indicate that cytoskeletal architecture has a significant influence on plant immunity, potentially controlled by both host and pathogen to alter the balance of resistance versus susceptibility.

Although the broader function and mechanisms associated with MF or MT reorganization in response to pathogen infection remain largely undefined, insight into the role of the cytoskeleton in plant immunity is becoming clearer through the analysis of individual MF- and MT-associated proteins. Among the first regulators of actin cytoskeletal organization revealed to play an important role in immunity are the actin depolymerizing factor (ADF) or cofilin (hereafter referred to as AC) family of proteins-a conserved class of small proteins that regulate actin cytoskeletal organization via filament severing and depolymerization (Kanellos and Frame 2016). As a family, AC are widely conserved across all eukaryotes, yet their abundance varies. In mammals, $3 \mathrm{AC}$ have been identified (i.e., ADF, CFL1, and CFL2) and, in most plants, dozens of ADF-encoding genes are present (11 in Arabidopsis and up to 27 in banana) (Kanellos and Frame 2016; Nan et al. 2017). Similar to their mammalian counterparts, plant ADF function as key regulators of cytoskeletal organization, controlling the overall balance of cellular G- and F-actin ratios.

In recent studies, ADF have also been shown to be associated with the function and activity of the plant immune system. For example, as a regulator of PTI, it was demonstrated that Arabidopsis ADF4 plays a key role in PAMP-triggered actin remodeling, demonstrating that $\mathrm{ADF} 4$ - and actin depolymerization-are necessary components of actin remodeling and callose deposition upon elf26 perception by the EFR (HentyRidilla et al. 2014). Moreover, in the case of fungal pathogen perception and immunity, the adf4 mutant was found to possess enhanced resistance, with subclass I ADF imparting an additive effect on pathogen susceptibility (Inada et al. 2016). These data suggest that resistance signaling associated with $\mathrm{ADF}$ function may, in fact, be mediated in a homolog- or class-specific manner; and, moreover, that expansion of the ADF gene family in plants, as compared with mammals, may impart roles for specific and individual ADF. Indeed, additional data support this hypothesis. Arabidopsis ADF6 was shown to negatively regulate the localization of RPW8.2 to extrahaustorial membranes to promote immune signaling (Wang et al. 2009); ADF3 is a positive regulator of resistance against aphids (Mondal et al. 2018); and, in wheat, TaADF4 and TaADF7 significantly contribute to resistance against the stripe rust pathogen $P u c$ cinia striiformis (Fu et al. 2014; Zhang et al. 2017) while $T a A D F 3$ is an negative regulator of this interaction (Tang et al. 2016).

In addition to ADF, the roles of other MF- or MT-associated proteins in plant immunity are beginning to emerge. For instance, capping protein which biochemically functions as a (+)-end actin polymerization inhibitor, is required for MF reorganization during immune signaling (Li et al. 2015; Li et al. 2017). Further, a recent study has shown that Arabidopsis Profilin3 (PFN3) negatively regulates PTI by inhibiting forminmediated actin polymerization (Sun et al. 2018). This discovery is interesting, because profilins are usually regarded as cofactors for formin-mediated actin polymerization. Thus, the biochemical function of AtPFN3 represents another strategy of cytoskeletal architecture regulation during immune activation-competitive inhibition of active cytoskeleton regulators. Converse to MF function during plant immunity, the molecular mechanisms of MT regulation during immune signaling events is relatively unclear. Thus, we posit that future work in the area of immune-MT interactions will lead to exciting new discoveries 
for the broader role of the cytoskeleton during plant defense and pathogen virulence.

\section{PATHOGEN TARGETING OF CYTOSKELETAL ORGANIZATION: IMMUNE SUBVERSION AND PATHOGENICITY}

Given the incredible connectivity of the cytoskeletal platform to nearly all cellular networks (Fig. 2), it is not surprising that pathogens and pests have evolved mechanisms to block immunity - either directly or indirectly — through manipulation of cytoskeletal function. In this respect, by targeting a few key steps in cytoskeletal assembly, for example, pathogens can gain access to a range of host mechanisms. To usurp, evade, or destroy? These are the evolved "choices" that pathogens have made to overtake the function and activity of the immune system at the cytoskeletal interface. In the case of plant viruses, whose amplification and intercellular movement require manipulation of the host cell machinery, including cytoskeleton (Hong and Ju 2017), the "choice" is to usurp. As a general strategy for viral manipulation of the cytoskeleton, the viral replication complex can load itself onto the cytoskeleton using scaffold proteins (e.g., movement protein or linking protein) or myosins, which enable the virus to track along the cytoskeletal network, including through plasmodesmata (Fig. 2 (j)) (Pitzalis and Heinlein 2017). As a result, the infecting virus is able to move from cell to cell, overwhelming immunity and ultimately taking control of the host.

As noted above, pathogen effector molecules function to subvert immune signaling and, in recent years, much effort has been spent on the discovery of the constellation of host processes targeted by these secreted factors. Thus, it was only a matter of time before pathogen effectors were identified which can directly or indirectly influence cytoskeletal function. In the case of indirect modulation of cytoskeletal function, work from Lee and colleagues (2012) observed that the Pseudomonas syringae DC3000 T3E HopE1 can bind to calmodulin, a process that leads to disassociation of the microtubule-associated protein 65-1 from the MT network, resulting in an increase in susceptibility to DC3000. In a similar mechanism, the Xanthomonas euvesicatoria T3E AvrBsT, and acetyltransferase, was shown to acetylate ACETYLATED INTERACTING PROTEIN 1, causing it to dissociate from MT and leading to a dampening of plant immunity (Cheong et al. 2014). Interestingly, it has also been demonstrated that pathogenic effectors can also influence the regulation of cytoskeletal function within and between organelles. As demonstrated by (Erickson and associate (2018), the X. campestris T3E XopL, an E3 ligase, suppresses plastid stromule formation induced by Agrobacterium spp.; it is hypothesized that XopL targets unknown MT-associated proteins, a conclusion based on the observation that a nonactive mutant of XopL loses such function but binds to MT.

Finally, and in work supported by independent studies that converged on similar pathogen virulence mechanisms, is the case of the P. syringae T3E HopG1. Previous work showed that HopG1 is a mitochondria-targeted effector that suppresses plant immunity (Block et al. 2010). In a bid to define virulence factors that target host cytoskeletal immune signaling, Shimono and associates (2016b) demonstrated that HopG1 interacts with Arabidopsis kinesin 7.4 (i.e., Kin7.4) (Moschou et al. 2016), a mitochondria-localized motor protein (Itoh et al. 2001) whose function is required for actin filament organization. During P. syringae DC3000 infection of Arabidopsis, HopG1 is delivered into the host cell and, subsequently, associates with Kin7.4, resulting in actin bundling and enhanced disease symptom development. This is exciting from the standpoint of pathogen targeting of the cytoskeleton and in the broader context of a role for actin in immunity. For example, if HopG1 and kinesin associate on the mitochondrial outer membrane, this might suggest a mechanism to inhibit the motor activity of kinesin, leading to an impediment in mitochondrial motion through the concerted action of both (i.e., MF and MT) cytoskeletal networks. This would then lead to a reduction in the energy needed to support cytoskeletal function and dynamism (Bartolák-Suki et al. 2017). However, if HopG1 and kinesin localize within the mitochondria itself, it would indicate a role for HopG1 in the disruption of kinesin function and a broader role of mitochondria as a signaling hub for immunity and cell death through actin filament remodeling. We tend to favor the latter, because evidence for such a role is supported by numerous studies demonstrating a function for the actin-mitochondrial network as a hub for the activation of apoptosis (Elmore 2007), a process associated with pathogen-induced senescence (Shimono et al. 2016b).

Converse to the examples highlighted above-whereby pathogens finesse cytoskeletal function to promote infection and disease - pathogens have also evolved virulence strategies to disrupt actin cytoskeletal dynamics in a more abrupt manner. In short, pathogens can paralyze host immunity by directly dissembling the cytoskeletal machinery. In one of the first examples of directed targeting of the plant cytoskeleton by a phytopathogen, work by Lee and colleagues (2012) uncovered a mechanism whereby the $P$. syringae T3E HopZ1a, an acetyltransferase, can modify and disrupt the MT network to interfere with MT-supported processes such as trafficking. More recently, work by Kang and associates (2014) showed that the $P$. syringae T3E HopW1 disrupts F-actin integrity by directly depolymerizing actin filaments during infection, a process resulting in blocks to protein cargo trafficking and endocytosis. Such strategies are also employed by viral pathogens, such as the case of the Cucumber mosaic virus movement protein, which can sever F-actin to increase the size exclusion limit of plasmodesmata, potentially accelerating the viral spread to adjacent cells (Su et al. 2010). Additionally, a new study demonstrates that root-knot nematodes secret an effector, Meloidogyne incognita PFN3, into host "giant cells" (the feeding structure) to inhibit host actin polymerization and cause higher susceptibility (Leelarasamee et al. 2018).

Although each of these examples clearly demonstrates that direct disruption of the MT or MF networks is a strategy to impede plant immunity, the question remains as to how the activities of these effectors are coordinately regulated, given that absolute disruption of plant MF or MT does not always lead to attenuated immune response, as mentioned above. One hypothesis is that additional signals are generated during infection that lead to proper regulation of effector-mediated cytoskeleton dissemble. This would, hypothetically, result in the specific modulation of effectors' activities at different key stages of the infection process, which leads to disruption of immune signaling. Such strategies have been characterized in the case of Salmonella infection of human cells, in which the coregulation of the T3E SipA and SipC, with opposing yet cooperative actin-polymerizing and -depolymerizing activities, renders the immune escape (McGhie et al. 2001, 2009).

\section{EMERGING THEMES AND FUTURE DIRECTIONS: THE PTI, ETI, CYTOSKELETAL FRONTIER}

The past two decades have witnessed the discovery of numerous mechanisms underpinning the linkages between the cytoskeleton and plant immune system (Fig. 3). Although a 
number of mechanisms remain to be defined, a framework is starting to emerge that demonstrates the roles of MF and MT in processes associated with PTI, ETI, and pathogen virulence. As a roadmap for future research in this area, we believe that the following topics will be key in further defining the cytoskeletonpathogen connection.

\section{Pathogen perception.}

How is plant immunity regulated by the PM-associated cytoskeleton? On the one hand, while PRR (e.g., FLS2) activation is not inhibited by disrupting F-actin, the ROS burst intensity and response time are altered. Given that RBOHD activation by PRR complexes does require a function association with the cytoskeleton, the most obvious hypothesis is that the PMassociated cytoskeleton potentially functions as a scaffold for PRR regulatory complexes. Additionally, it will be important to determine whether the PM-associated cytoskeleton influences ETI through CC-NLR ETI activation. On the other hand, although the PM nanodomain containing MT-associated receptor complexes (BRI-BIK1) has been identified, the FLS2-BIK1 nanodomain has not been conclusively demonstrated to interact with MT using similar experimental approaches. This may indicate that different PM receptor complexes interact with different types of the cytoskeleton in a highly selective pattern. These mechanisms in plants, if revealed, may parallel what is currently described in innate immune signaling processes in animals, and provide new engineering strategies to optimize plant immunity.
Signaling-reorganization pattern specificity.

What is the common and specific pattern of cytoskeleton reorganization by different types immune signaling and what is the mechanism beyond? As mentioned above, multiple types of immune signaling-PTI and ETI-can trigger the reorganization of both MF and MT. However, recent data demonstrate that the nature, duration, and amplitude of these reorganization events is dependent upon the elicitor itself. To date, though some limited high-resolution data of cytoskeleton reorganization triggered by various signals have been obtained, there is still a knowledge gap between the observed phenotypes and their molecular mechanism, including the biological significance of these responses. Further work in this area will not only address these gaps but also likely lead to a better understanding of the role of actin in PTI and ETI, including points of convergence and divergence.

\section{Regulation and pathogen targeting of the plant cytoskeleton.}

What is the signaling pathway from PRR activation to resultant cytoskeleton reorganization? An abundance of data supports the critical role for ADF and other actin binding proteins (ABPs) in immunity against plant pathogens. However, knowledge gaps remain as to (i) how the activities of these proteins are regulated in plants, (ii) the biological and biochemical significance of such regulation and (iii) how pathogens target host cytoskeleton regulatory mechanisms,

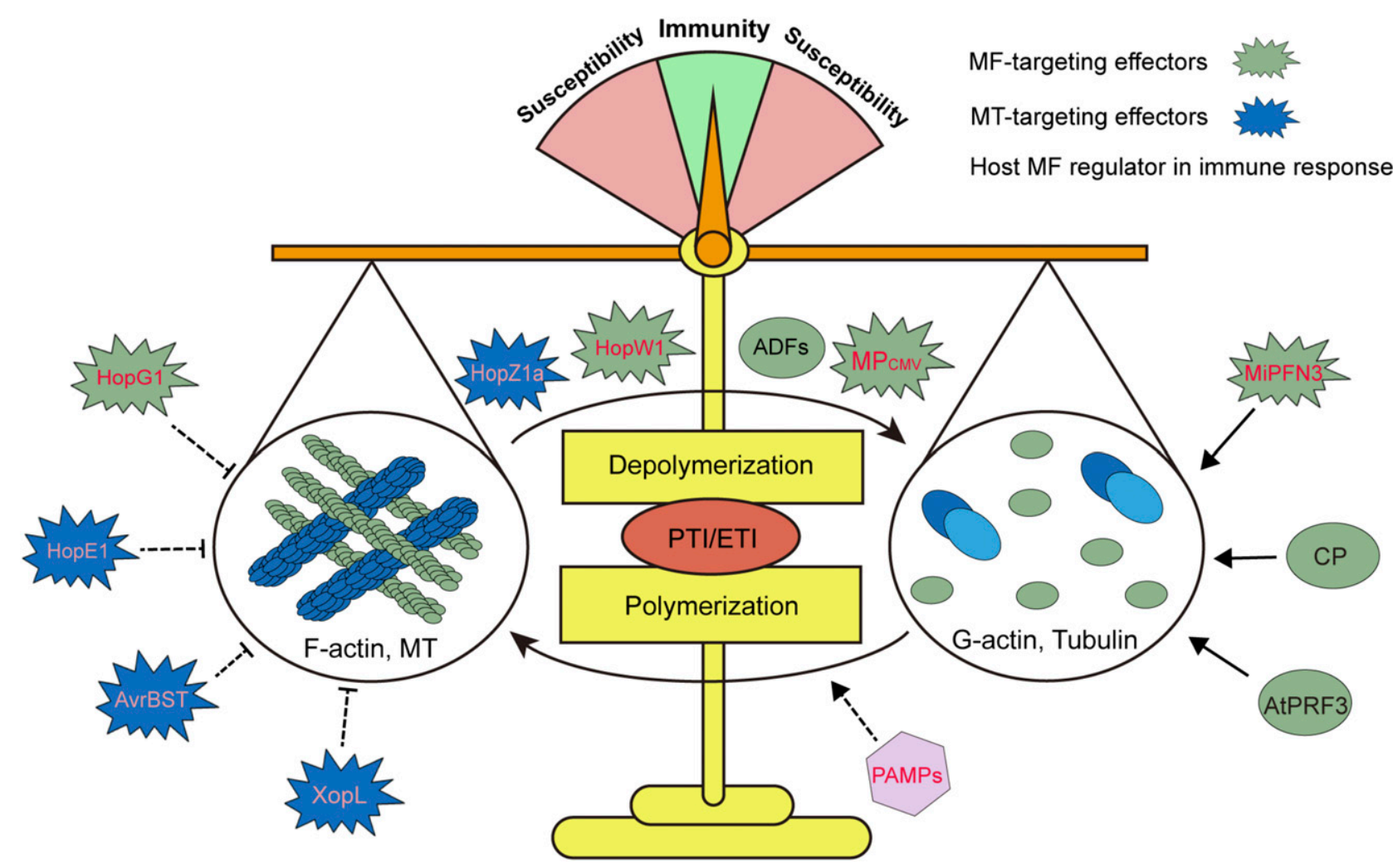

Fig. 3. The cytoskeleton is central to the balance between immunity and susceptibility, and the host-dominated balance and alterability between G-actin, Factin, and tubulin control the plant response to pests and pathogens. The cytoskeleton is tightly regulated by the temporal and spatial control of filament architecture, and these points of control are influenced by the perception of pathogens and pathogen elicitors (i.e., pathogen-associated molecular pattern [PAMP] and effectors). For the host, identified actin regulators in immune response includes ADF (depolymerizing and severing actin), capping protein (stabilizing short F-actin oligo and G-actin), and AtPFR3 (stabilizing G-actin). Pathogens and pests, on the other hand, can use effectors to interfere the host regulation of cytoskeleton. For instance, HopW1 and the Cucumber mosaic virus movement protein (MP) can directly sever F-actin, thus increasing the cellular concentration of G-actin. Similarly, HopZ1a can disrupt microtubule (MT) filaments by acetylating tubulins, a process that results in disruption of the MT network and associated process. In the case of microfilament (MF) function, Meloidogyne incognita Profilin3 can stabilize G-actin and directly inhibit actin assembly. HopG1, HopE1, AvrBsT, and XopL can indirectly interfere with host cytoskeletal function. PTI = PAMP-triggered immunity and ETI = effectortriggered immunity. 
thus leading to misregulation of the cytoskeleton and further blocking of immune signaling and host defense. Further, the pattern of MT reorganization in response to immune signaling, and the proteins participating in such regulation, are even less defined than those of MF activity. It is anticipated that additional participants of cytoskeletal regulation during immune events will be revealed.

\section{From the outside, in.}

What is the function of the actin system inside the nucleus? The extraordinary connectivity of the cytoskeleton gives the cell unfettered access to a range of processes and environments. Further definition of how signals are transduced from the PM to the nucleus will provide insight into the surveillance and regulatory functions of the immune system as a continuum, from the periphery of the cell to the nucleus. In mammal systems, this process is well documented (Dopie et al. 2012; Stüven et al. 2003; Wada et al. 1998). Similar to the operation of the plant immune system, the nuclearcytoplasmic shuttling of actin and ABP is hypothesized to maintain an active and highly responsive surveillance platform (Fig. $2(\mathrm{k})$ ). In plants, preliminary studies have uncovered a role for nuclear-cytoplasmic shuttling of actin during pathogenesis (Levy et al. 2013), and nuclear ABP such as ADF (Inada et al. 2016) have an impact on the plant immune response. Thus, the framework exists to further define the processes described here toward extending our understanding of the role of the cytoskeleton in almost every step of plant immune response, from pathogen perception to the regulation of the immune transcriptome.

\section{ACKNOWLEDGMENTS}

We thank the members of the Day laboratory and N. Day for useful comments and feedback during the preparation of this manuscript.

\section{LITERATURE CITED}

Bacete, L., Mélida, H., Miedes, E., and Molina, A. 2018. Plant cell wallmediated immunity: Cell wall changes trigger disease resistance responses. Plant J. 93:614-636.

Bartolák-Suki, E., Imsirovic, J., Nishibori, Y., Krishnan, R., and Suki, B. 2017. Regulation of mitochondrial structure and dynamics by the cytoskeleton and mechanical factors. Int. J. Mol. Sci. 18:1812.

Beck, M., Zhou, J., Faulkner, C., MacLean, D., and Robatzek, S. 2012. Spatio-temporal cellular dynamics of the Arabidopsis flagellin receptor reveal activation status-dependent endosomal sorting. Plant Cell 24: 4205-4219.

Bielig, H., Lautz, K., Braun, P. R., Menning, M., Machuy, N., Brügmann, C., Barisic, S., Eisler, S. A., Andree, M., Zurek, B., Kashkar, H., Sansonetti, P. J., Hausser, A., Meyer, T. F., and Kufer, T. A. 2014. The cofilin phosphatase slingshot homolog 1 (SSH1) links NOD1 signaling to actin remodeling. PLoS Pathog. 10:e1004351.

Block, A., Guo, M., Li, G., Elowsky, C., Clemente, T. E., and Alfano, J. R. 2010. The Pseudomonas syringae type III effector HopG1 targets mitochondria, alters plant development and suppresses plant innate immunity. Cell. Microbiol. 12:318-330.

Brandizzi, F., and Wasteneys, G. O. 2013. Cytoskeleton-dependent endomembrane organization in plant cells: An emerging role for microtubules. Plant J. 75:339-349.

Bücherl, C. A., Jarsch, I. K., Schudoma, C., Segonzac, C., Mbengue, M., Robatzek, S., MacLean, D., Ott, T., and Zipfel, C. 2017. Plant immune and growth receptors share common signalling components but localise to distinct plasma membrane nanodomains. eLife 6:e25114.

Büttner, D. 2016. Behind the lines-actions of bacterial type III effector proteins in plant cells. FEMS Microbiol. Rev. 40:894-937.

Cai, G., Faleri, C., Del Casino, C., Emons, A. M., and Cresti, M. 2011. Distribution of callose synthase, cellulose synthase, and sucrose synthase in tobacco pollen tube is controlled in dissimilar ways by actin filaments and microtubules. Plant Physiol. 155:1169-1190.
Caplan, J. L., Kumar, A. S., Park, E., Padmanabhan, M. S., Hoban, K. Modla, S., Czymmek, K., and Dinesh-Kumar, S. P. 2015. Chloroplast stromules function during innate immunity. Dev. Cell 34:45-57.

Caplan, J. L., Mamillapalli, P., Burch-Smith, T. M., Czymmek, K., and Dinesh-Kumar, S. P. 2008. Chloroplastic protein NRIP1 mediates innate immune receptor recognition of a viral effector. Cell 132:449-462.

Cheong, M. S., Kirik, A., Kim, J. G., Frame, K., Kirik, V., and Mudgett, M. B. 2014. AvrBsT acetylates Arabidopsis ACIP1, a protein that associates with microtubules and is required for immunity. PLoS Pathog. 10:e1003952.

Chisholm, S. T., Coaker, G., Day, B., and Staskawicz, B. J. 2006. Hostmicrobe interactions: Shaping the evolution of the plant immune response. Cell 124:803-814.

Couto, D., and Zipfel, C. 2016. Regulation of pattern recognition receptor signalling in plants. Nat. Rev. Immunol. 16:537-552.

Day, B., Henty, J. L., Porter, K. J., and Staiger, C. J. 2011. The pathogenactin connection: A platform for defense signaling in plants. Annu. Rev. Phytopathol. 49:483-506

Dong, J., and Chen, W. 2013. The role of autophagy in chloroplast degradation and chlorophagy in immune defenses during Pst DC3000 (AvrRps4) infection. PLoS One 8:e73091.

Dopie, J., Skarp, K.-P., Rajakylä, E. K., Tanhuanpää, K., and Vartiainen, M. K. 2012. Active maintenance of nuclear actin by importin 9 supports transcription. Proc. Natl. Acad. Sci. U.S.A. 109:E544-E552.

Elliott, A., and Shaw, S. L. 2018. Update: Plant cortical microtubule arrays. Plant Physiol. 176:94-105.

Elmore, S. 2007. Apoptosis: A review of programmed cell death. Toxicol. Pathol. 35:495-516.

Erickson, J. L., Adlung, N., Lampe, C., Bonas, U., and Schattat, M. H. 2018. The Xanthomonas effector XopL uncovers the role of microtubules in stromule extension and dynamics in Nicotiana benthamiana. Plant J. 93:856-870.

Fan, L., Li, R., Pan, J., Ding, Z., and Lin, J. 2015. Endocytosis and its regulation in plants. Trends Plant Sci. 20:388-397.

Fu, Y., Duan, X., Tang, C., Li, X., Voegele, R. T., Wang, X., Wei, G., and Kang, Z. 2014. TaADF7, an actin-depolymerizing factor, contributes to wheat resistance against Puccinia strifformis f. sp. tritici. Plant J. 78 16-30.

Guan, X., Buchholz, G., and Nick, P. 2013. The cytoskeleton is disrupted by the bacterial effector HrpZ, but not by the bacterial PAMP flg22, in tobacco BY-2 cells. J. Exp. Bot. 64:1805-1816.

Hanson, M. R., and Hines, K. M. 2018. Stromules: Probing formation and function. Plant Physiol. 176:128-137.

He, Z., Huang, T., Ao, K., Yan, X., and Huang, Y. 2017. Sumoylation, phosphorylation, and acetylation fine-tune the turnover of plant immunity components mediated by ubiquitination. Front. Plant Sci. 8 : 1682.

Henty-Ridilla, J. L., Li, J., Day, B., and Staiger, C. J. 2014. ACTIN DEPOLYMERIZING FACTOR4 regulates actin dynamics during innate immune signaling in Arabidopsis. Plant Cell 26:340-352.

Henty-Ridilla, J. L., Shimono, M., Li, J., Chang, J. H., Day, B., and Staiger, C. J. 2013. The plant actin cytoskeleton responds to signals from microbe-associated molecular patterns. PLoS Pathog. 9:e1003290.

Hong, J.-S., and Ju, H.-J. 2017. The plant cellular systems for plant virus movement. Plant Pathol. J. 33:213-228.

Huot, B., Yao, J., Montgomery, B. L., and He, S. Y. 2014. Growth-defense tradeoffs in plants: A balancing act to optimize fitness. Mol. Plant 7: 1267-1287.

Huysmans, M., Lema A, S., Coll, N. S., and Nowack, M. K. 2017. Dying two deaths-Programmed cell death regulation in development and disease. Curr. Opin. Plant Biol. 35:37-44.

Inada, N., Higaki, T., and Hasezawa, S. 2016. Nuclear function of subclass I actin-depolymerizing factor contributes to susceptibility in Arabidopsis to an adapted powdery mildew fungus. Plant Physiol. 170:1420-1434.

Itoh, R., Fujiwara, M., and Yoshida, S. 2001. Kinesin-related proteins with a mitochondrial targeting signal. Plant Physiol. 127:724-726.

Jones, J. D., and Dangl, J. L. 2006. The plant immune system. Nature 444:323-329.

Kaksonen, M., and Roux, A. 2018. Mechanisms of clathrin-mediated endocytosis. Nat. Rev. Mol. Cell Biol. 19:313-326.

Kanellos, G., and Frame, M. C. 2016. Cellular functions of the ADF/cofilin family at a glance. J. Cell Sci. 129:3211-3218.

Kang, Y., Jelenska, J., Cecchini, N. M., Li, Y., Lee, M. W., Kovar, D. R., and Greenberg, J. T. 2014. HopW1 from Pseudomonas syringae disrupts the actin cytoskeleton to promote virulence in Arabidopsis. PLoS Pathog. 10:e1004232.

Keinath, N. F., Kierszniowska, S., Lorek, J., Bourdais, G., Kessler, S. A., Shimosato-Asano, H., Grossniklaus, U., Schulze, W. X., Robatzek, S. 
and Panstruga, R. 2010. PAMP (pathogen-associated molecular pattern)induced changes in plasma membrane compartmentalization reveal novel components of plant immunity. J. Biol. Chem. 285:39140-39149.

Kobayashi, I., Kobayashi, Y., Yamaoka, N., and Kunoh, H. 1992. Recognition of a pathogen and a nonpathogen by barley coleoptile cells. III. Responses of microtubules and actin filaments in barley coleoptile cells to penetration attempts. Can. J. Bot. 70:1815-1823.

Krutinova, H., Trda, L., Kalachova, T., Lamparova, L., Pospichalova, R., Dobrev, P., Malinska, K., Burketova, L., Valentova, O., and Janda, M. 2018. Can actin depolymerization actually result In increased plant resistance to pathogens? Online publication. bioRxiv

Kufer, T. A., Kremmer, E., Adam, A. C., Philpott, D. J., and Sansonetti, P. J. 2008. The pattern-recognition molecule Nod1 is localized at the plasma membrane at sites of bacterial interaction. Cell. Microbiol. 10:477-486.

Kumar, A. S., Park, E., Nedo, A., Alqarni, A., Ren, L., Hoban, K., Modla, S., McDonald, J. H., Kambhamettu, C., Dinesh-Kumar, S. P., and Caplan, J. L. 2018. Stromule extension along microtubules coordinated with actin-mediated anchoring guides perinuclear chloroplast movement during innate immunity. eLife 7:e23625.

Lee, A. H. Y., Hurley, B., Felsensteiner, C., Yea, C., Ckurshumova, W., Bartetzko, V., Wang, P. W., Quach, V., Lewis, J. D., Liu, Y. C., Börnke, F., Angers, S., Wilde, A., Guttman, D. S., and Desveaux, D. 2012. A bacterial acetyltransferase destroys plant microtubule networks and blocks secretion. PLoS Pathog. 8:e1002523.

Leelarasamee, N., Zhang, L., and Gleason, C. 2018. The root-knot nematode effector MiPFN3 disrupts plant actin filaments and promotes parasitism. PLoS Pathog. 14:e1006947.

Legrand-Poels, S., Kustermans, G., Bex, F., Kremmer, E., Kufer, T. A., and Piette, J. 2007. Modulation of Nod2-dependent NF- $\kappa B$ signaling by the actin cytoskeleton. J. Cell Sci. 120:1299-1310.

Levy, A., Zheng, J. Y., and Lazarowitz, S. G. 2013. The tobamovirus Turnip Vein Clearing Virus 30-kilodalton movement protein localizes to novel nuclear filaments to enhance virus infection. J. Virol. 87:6428-6440.

Li, B., Meng, X., Shan, L., and He, P. 2016. Transcriptional regulation of pattern-triggered immunity in plants. Cell Host Microbe 19:641-650.

Li, J., Cao, L., and Staiger, C. J. 2017. Capping protein modulates actin remodeling in response to reactive oxygen species during plant innate immunity. Plant Physiol. 173:1125-1136.

Li, J., Henty-Ridilla, J. L., Staiger, B. H., Day, B., and Staiger, C. J. 2015. Capping protein integrates multiple MAMP signalling pathways to modulate actin dynamics during plant innate immunity. Nat. Commun. 6: Article 7206

Li, S., Blanchoin, L., Yang, Z., and Lord, E. M. 2003. The putative Arabidopsis arp2/3 complex controls leaf cell morphogenesis. Plant Physiol. 132:2034-2044.

Lo Presti, L., and Kahmann, R. 2017. How filamentous plant pathogen effectors are translocated to host cells. Curr. Opin. Plant Biol. 38:19-24.

Luna, E., Pastor, V., Robert, J., Flors, V., Mauch-Mani, B., and Ton, J. 2011. Callose deposition: A multifaceted plant defense response. Mol. PlantMicrobe Interact. 24:183-193.

Mbengue, M., Bourdais, G., Gervasi, F., Beck, M., Zhou, J., Spallek, T., Bartels, S., Boller, T., Ueda, T., Kuhn, H., and Robatzek, S. 2016. Clathrin-dependent endocytosis is required for immunity mediated by pattern recognition receptor kinases. Proc. Natl. Acad. Sci. U.S.A. 113: 11034-11039.

McGhie, E. J., Brawn, L. C., Hume, P. J., Humphreys, D., and Koronakis, V. 2009. Salmonella takes control: Effector-driven manipulation of the host. Curr. Opin. Microbiol. 12:117-124.

McGhie, E. J., Hayward, R. D., and Koronakis, V. 2001. Cooperation between actin-binding proteins of invasive Salmonella: SipA potentiates SipC nucleation and bundling of actin. EMBO J. 20:2131-2139.

Miklis, M., Consonni, C., Bhat, R. A., Lipka, V., Schulze-Lefert, P., and Panstruga, R. 2007. Barley MLO modulates actin-dependent and actinindependent antifungal defense pathways at the cell periphery. Plant Physiol. 144:1132-1143.

Mondal, H. A., Louis, J., Archer, L., Patel, M., Nalam, V. J., Sarowar, S., Sivapalan, V., Root, D. D., and Shah, J. 2018. Arabidopsis ACTINDEPOLYMERIZING FACTOR3 is required for controlling aphid feeding from the phloem. Plant Physiol. 176:879-890.

Moschou, P. N., Gutierrez-Beltran, E., Bozhkov, P. V., and Smertenko, A 2016. Separase promotes microtubule polymerization by activating CENP-E-related kinesin Kin7. Dev. Cell 37:350-361.

Nagawa, S., Xu, T., Lin, D., Dhonukshe, P., Zhang, X., Friml, J., Scheres, B., Fu, Y., and Yang, Z. 2012. ROP GTPase-dependent actin microfilaments promote PIN1 polarization by localized inhibition of clathrin-dependent endocytosis. PLoS Biol. 10:e1001299.

Nan, Q., Qian, D., Niu, Y., He, Y., Tong, S., Niu, Z., Ma, J., Yang, Y., An, L., Wan, D., and Xiang, Y. 2017. Plant actin-depolymerizing factors possess opposing biochemical properties arising from key amino acid changes throughout evolution. Plant Cell 29:395-408.

Natesan, S. K., Sullivan, J. A., and Gray, J. C. 2009. Myosin XI is required for actin-associated movement of plastid stromules. Mol. Plant 2: 1262-1272.

Nebenführ, A., and Dixit, R. 2018. Kinesins and myosins: Molecular motors that coordinate cellular functions in plants. Annu. Rev. Plant Biol. 69:329-361.

Opalski, K. S., Schultheiss, H., Kogel, K. H., and Hückelhoven, R. 2005. The receptor-like MLO protein and the RAC/ROP family G-protein RACB modulate actin reorganization in barley attacked by the biotrophic powdery mildew fungus Blumeria graminis f. sp. hordei. Plant J. 41:291-303.

Ortiz-Morea, F. A., Savatin, D. V., Dejonghe, W., Kumar, R., Luo, Y., Adamowski, M., Van den Begin, J., Dressano, K., Pereira de Oliveira, G., Zhao, X., Lu, Q., Madder, A., Friml, J., Scherer de Moura, D. and Russinova, E. 2016. Danger-associated peptide signaling in Arabidopsis requires clathrin. Proc. Natl. Acad. Sci. U.S.A. 113 11028-11033.

Park, E., Nedo, A., Caplan, J. L., and Dinesh-Kumar, S. P. 2018. Plantmicrobe interactions: Organelles and the cytoskeleton in action. New Phytol. 217:1012-1028.

Peng, Y., van Wersch, R., and Zhang, Y. 2018. Convergent and divergent signaling in PAMP-triggered immunity and effector-triggered immunity Mol. Plant-Microbe Interact.31:403-409.

Pitzalis, N., and Heinlein, M. 2017. The roles of membranes and associated cytoskeleton in plant virus replication and cell-to-cell movement. J. Exp. Bot. 69:117-132.

Porter, K., and Day, B. 2016. From filaments to function: The role of the plant actin cytoskeleton in pathogen perception, signaling and immunity. J. Integr. Plant Biol. 58:299-311.

Postma, J., Liebrand, T. W., Bi, G., Evrard, A., Bye, R. R., Mbengue, M., Kuhn, H., Joosten, M. H., and Robatzek, S. 2016. Avr4 promotes Cf-4 receptor-like protein association with the BAK1/SERK3 receptor-like kinase to initiate receptor endocytosis and plant immunity. New Phytol. 210:627-642

Schmidt, S. M., and Panstruga, R. 2007. Cytoskeleton functions in plant-microbe interactions. Physiol. Mol. Plant Pathol. 71:135-148.

Schneider, R., Hanak, T., Persson, S., and Voigt, C. A. 2016. Cellulose and callose synthesis and organization in focus, what's new? Curr. Opin Plant Biol. 34:9-16.

Shimono, M., Higaki, T., Kaku, H., Shibuya, N., Hasezawa, S., and Day, B. 2016a. Quantitative evaluation of stomatal cytoskeletal patterns during the activation of immune signaling in Arabidopsis thaliana. PLoS One 11:e0159291.

Shimono, M., Lu, Y. J., Porter, K., Kvitko, B. H., Henty-Ridilla, J., Creason, A., He, S. Y., Chang, J. H., Staiger, C. J., and Day, B. 2016b. The Pseudomonas syringae type III effector HopG1 induces actin remodeling to promote symptom development and susceptibility during infection. Plant Physiol. 171:2239-2255.

Skalamera, D., and Heath, M. C. 1998. Changes in the cytoskeleton accompanying infection-induced nuclear movements and the hypersensitive response in plant cells invaded by rust fungi. Plant J. 16:191-200.

Stüven, T., Hartmann, E., and Görlich, D. 2003. Exportin 6: A novel nuclear export receptor that is specific for profilin - actin complexes. EMBO J. 22:5928-5940.

Su, J., Spears, B. J., Kim, S. H., and Gassmann, W. 2018. Constant vigilance: Plant functions guarded by resistance proteins. Plant J. 93: 637-650.

Su, S., Liu, Z., Chen, C., Zhang, Y., Wang, X., Zhu, L., Miao, L., Wang, X.-C., and Yuan, M. 2010. Cucumber mosaic virus movement protein severs actin filaments to increase the plasmodesmal size exclusion limit in tobacco. Plant Cell 22:1373-1387.

Sun, H., Qiao, Z., Chua, K. P., Tursic, A., Liu, X., Gao, Y. G., Mu, Y., Hou, X., and Miao, Y. 2018. Profilin negatively regulates formin-mediated actin assembly to modulate PAMP-triggered plant immunity. Curr. Biol. 28:1882-1895.e7.

Takemoto, D., Jones, D. A., and Hardham, A. R. 2003. GFP-tagging of cell components reveals the dynamics of subcellular re-organization in response to infection of Arabidopsis by oomycete pathogens. Plant J. 33: 775-792.

Takemoto, D., Jones, D. A., and Hardham, A. R. 2006. Re-organization of the cytoskeleton and endoplasmic reticulum in the Arabidopsis pen1-1 mutant inoculated with the non-adapted powdery mildew pathogen, Blumeria graminis f. sp. hordei. Mol. Plant Pathol. 7:553-563.

Tang, C., Deng, L., Chang, D., Chen, S., Wang, X., and Kang, Z. 2016. TaADF3, an actin-depolymerizing factor, negatively modulates 
wheat resistance against Puccinia striiformis. Front. Plant Sci. 6: 1214.

Tang, D., Wang, G., and Zhou, J. M. 2017. Receptor kinases in plantpathogen interactions: More than pattern recognition. Plant Cell 29: 618-637.

Tian, M., Chaudhry, F., Ruzicka, D. R., Meagher, R. B., Staiger, C. J., and Day, B. 2009. Arabidopsis actin-depolymerizing factor AtADF4 mediates defense signal transduction triggered by the Pseudomonas syringae effector AvrPphB. Plant Physiol. 150: 815-824.

Tominaga, M., and Ito, K. 2015. The molecular mechanism and physiological role of cytoplasmic streaming. Curr. Opin. Plant Biol. 27:104-110.

Tóth, K., and Stacey, G. 2015. Does plant immunity play a critical role during initiation of the legume-rhizobium symbiosis? Front. Plant Sci. 6:401.

van de Meene, A. M., Doblin, M. S., and Bacic, A. 2017. The plant secretory pathway seen through the lens of the cell wall. Protoplasma 254:75-94.

Wada, A., Fukuda, M., Mishima, M., and Nishida, E. 1998. Nuclear export of actin: A novel mechanism regulating the subcellular localization of a major cytoskeletal protein. EMBO J. 17:1635-1641.
Wang, W., Wen, Y., Berkey, R., and Xiao, S. 2009. Specific targeting of the Arabidopsis resistance protein RPW8.2 to the interfacial membrane encasing the fungal haustorium renders broad-spectrum resistance to powdery mildew. Plant Cell 21:2898-2913.

Wang, Y., Zheng, X., Yu, B., Han, S., Guo, J., Tang, H., Yu, A. Y., Deng, H., Hong, Y., and Liu, Y. 2015. Disruption of microtubules in plants suppresses macroautophagy and triggers starch excess-associated chloroplast autophagy. Autophagy 11:2259-2274.

Yang, L., Qin, L., Liu, G., Peremyslov, V. V., Dolja, V. V., and Wei, Y. 2014. Myosins XI modulate host cellular responses and penetration resistance to fungal pathogens. Proc. Natl. Acad. Sci. U.S.A. 111:13996-14001.

Yi, S. Y., Shirasu, K., Moon, J. S., Lee, S. G., and Kwon, S. Y. 2014. The activated SA and JA signaling pathways have an influence on flg22triggered oxidative burst and callose deposition. PLoS One 9:e88951.

Zhang, B., Hua, Y., Wang, J., Huo, Y., Shimono, M., Day, B., and Ma, Q. 2017. TaADF4, an actin-depolymerizing factor from wheat, is required for resistance to the stripe rust pathogen Puccinia striiformis $\mathrm{f}$. sp. tritici. Plant J. 89:1210-1224. 